\title{
Weak and Strong Convergence Theorems for Finite Families of Asymptotically Quasi-Nonexpansive Mappings in Banach Spaces
}

\author{
Lei Deng and Juan Xiao \\ School of Mathematics and Statistics, Southwest University, Chongqing 400715, China \\ Correspondence should be addressed to Lei Deng; denglei@swu.edu.cn
}

Received 6 February 2014; Accepted 14 May 2014; Published 27 May 2014

Academic Editor: Nan-Jing Huang

Copyright (C) 2014 L. Deng and J. Xiao. This is an open access article distributed under the Creative Commons Attribution License, which permits unrestricted use, distribution, and reproduction in any medium, provided the original work is properly cited.

A finite-step iteration sequence for two finite families of asymptotically nonexpansive mappings is introduced and the weak and strong convergence theorems are proved in Banach space. The results presented in the paper generalize and unify some important known results of relevant scholars.

\section{Introduction and Preliminaries}

Throughout this work, we assume that $E$ is a real Banach space and $K$ is a nonempty subset of $E$. A mapping $T: C \rightarrow$ $C$ is said to be asymptotically nonexpansive if there exists a sequence $\left\{k_{n}\right\} \subset[1, \infty)$ with $\lim _{n \rightarrow \infty} k_{n}=1$ such that

$$
\left\|T^{n} x-T^{n} y\right\| \leq k_{n}\|x-y\|, \quad \forall x, y \in K, \forall n \geq 1 .
$$

The class of asymptotically nonexpansive mappings was introduced by Goebel and Kirk [1] in 1972 as an important generalization of the class of nonexpansive self-mappings, who proved that if $K$ is a nonempty closed convex subset of a real uniformly convex Banach space and $T$ is an asymptotically nonexpansive self-mapping of $K$, then $T$ has a fixed point. Strong and weak convergence theorems for nonexpansive and asymptotically nonexpansive families of mappings and for single maps have been established by many authors (see [2-11]).

In [2], the authors introduced a multistep procedure defined by (2); under some conditions, they proved that the convergence of Mann-Ishikawa iterations is equivalent to the convergence of the multistep iteration in Banach spaces:

$$
\begin{gathered}
x_{1} \in E, \\
x_{n+1}=\left(1-\beta_{n}^{1}\right) x_{n}+\beta_{n}^{1} T y_{n}^{1},
\end{gathered}
$$

$$
\begin{gathered}
y_{n}^{i}=\left(1-\beta_{n}^{i+1}\right) x_{n}+\beta_{n}^{i+1} T y_{n}^{i+1}, \quad i=1,2, \ldots, m-2, \\
y_{n}^{m-1}=\left(1-\beta_{n}^{m}\right) x_{n}+\beta_{n}^{m} T x_{n}, \quad m \geq 2,
\end{gathered}
$$

where the sequences $\left\{\beta_{n}^{i}\right\}_{n=1}^{\infty} \subseteq[0,1], i=1,2, \ldots, m$ satisfy certain conditions.

In [3], Chidume and Ali studied a scheme defined by

$$
\begin{gathered}
x_{1} \in K, \\
x_{n+1}=\left(1-\beta_{n}^{1}\right) x_{n}+\beta_{n}^{1} T_{1}^{n} y_{n+m-2}, \\
y_{n+m-2}=\left(1-\beta_{n}^{2}\right) x_{n}+\beta_{n}^{2} T_{2}^{n} y_{n+m-3}, \quad n \geq 1, \\
\vdots \\
y_{n}=\left(1-\beta_{n}^{m}\right) x_{n}+\beta_{n}^{m} T_{m}^{n} x_{n}, \quad m \geq 2,
\end{gathered}
$$

where $\left\{\beta_{n}^{i}\right\}_{i=1}^{m}$ is a sequence in $[\epsilon, 1-\epsilon], \epsilon \in(0,1)$. In a real uniformly convex Banach space $E$, they proved the following: (i) a weak convergence theorem for finite families of asymptotically nonexpansive mappings where the dual space $E^{*}$ of $E$ satisfies the Kadec-Klee property; (ii) a strong convergence theorem if one member of the family of asymptotically nonexpansive maps $\left\{T_{i}\right\}$ satisfies a condition weaker than semicompactness. 
Now, a finite-step iteration sequence for two finite families of asymptotically nonexpansive mappings is introduced as follows.

Let $K$ be a nonempty closed convex subset of a Banach space $E$, and let $\left\{T_{i}\right\}_{i=1}^{m},\left\{S_{i}\right\}_{i=1}^{m}: K \rightarrow K$ be two finite families of asymptotically quasi-nonexpansive mappings; the iterative sequence $\left\{x_{n}\right\}$ is defined by the iterative scheme

$$
\begin{gathered}
x_{j} \in E \quad(j=1,2, \ldots, r), \\
x_{n+1}=\left(1-\beta_{n}^{1}\right) S_{1}^{n} x_{n}+\beta_{n}^{1} T_{1}^{n} y_{n-q_{1}}^{1}, \\
y_{n-q_{i}}^{i}=\left(1-\beta_{n}^{i+1}\right) S_{i+1}^{n} x_{n}+\beta_{n}^{i+1} T_{i+1}^{n} y_{n-q_{i+1}}^{i+1}, \\
i=1,2, \ldots, m-2, \\
y_{n-q_{m-1}}^{m-1}=\left(1-\beta_{n}^{m}\right) S_{m}^{n} x_{n}+\beta_{n}^{m} T_{m}^{n} x_{n}, \\
m \geq 2, n \geq r,
\end{gathered}
$$

where $\left\{\beta_{n}^{i}\right\}_{i=1}^{m} \subset[\epsilon, 1-\epsilon]$ with $\epsilon \in(0,1),\left\{q_{i}\right\}_{i=1}^{m}$ is a nonnegative integer sequence in $[0, r)$, and $r, m \in \mathbb{N}$ are fixed numbers.

Remark 1. In (4), taking $r=1, T_{i}=T, S_{i}=I$, and $q_{i}=0$ for all $i=1,2, \ldots, m$, then we obtain (2); taking $r=1, S_{i}=I$, $y_{n-q_{i+1}}^{i+1}=y_{n+m-i+2}$, and $q_{i}=0$ for all $i=1,2, \ldots, m$, then we obtain (3).

In this paper, the finite families of asymptotically quasinonexpansive mappings are defined in Banach spaces. Under certain conditions, we construct an iterative scheme and prove the following: (i) a weak convergence theorem for finite families of asymptotically quasi-nonexpansive mappings, where the uniformly convex Banach space satisfies Opial's condition; (ii) necessary and sufficient conditions for convergence in real Banach spaces and a strong convergence theorem if the finite families of asymptotically quasi-nonexpansive mappings satisfy condition $(B)$. Our results generalize and unify many important known results of relevant scholars.

In order to prove the main results of this work, we need some basic concepts indicated as follows.

Let $E$ be a Banach space, and let $K$ be a nonempty closed convex subset of a $E$. A mapping $T$ with domain $D(T)$ and range $R(T)$ in $E$ is said to be demiclosed at $p$ [3] if whenever $\left\{x_{n}\right\}$ is a sequence in $D(T)$ such that $x_{n} \rightarrow x^{*} \in D(T)$ and $T x_{n} \rightarrow p$, then $T x^{*}=p$.

$E$ is said to satisfy Opial's condition [5] if, for any sequence $x_{n} \in E, x_{n} \rightarrow x$ implies that $\limsup _{n \rightarrow \infty}\left\|x_{n}-x\right\|<$ $\lim \sup _{n \rightarrow \infty}\left\|x_{n}-y\right\|$ for all $y \in E$ with $y \neq x$, where $x_{n} \rightarrow x$ denotes that $\left\{x_{n}\right\}$ converges weakly to $x$.

Let $\left\{T_{i}\right\}_{i=1}^{m}$ be the self-mappings of $K$ and $F\left(T_{i}\right)$ denotes the set of fixed points of $T_{i}$.

Definition 2. $\left\{T_{i}\right\}_{i=1}^{m}$ is said to be a finite family of asymptotically nonexpansive mappings if there exists a sequence $u_{n} \in$ $[0, \infty), \lim _{n \rightarrow \infty} u_{n}=0$, such that $\left\|T_{i}^{n} x-T_{i}^{n} y\right\| \leq\left(1+u_{n}\right)\|x-y\|$ for all $n \in \mathbb{N}$ and $x, y \in K$.
Definition 3. $\left\{T_{i}\right\}_{i=1}^{m}$ is called a finite family of asymptotically quasi-nonexpansive mappings if there exists a sequence $u_{n} \epsilon$ $[0, \infty), \lim _{n \rightarrow \infty} u_{n}=0$, such that $\left\|T_{i}^{n} x-p\right\| \leq\left(1+u_{n}\right)\|x-p\|$ for all $n \in \mathbb{N}$, and $x \in K, p \in F(T)$, where $F(T) \neq \varnothing$.

Remark 4 . The class of asymptotically quasi-nonexpansive mappings is a generalization of the class of nonexpansive mappings and asymptotically nonexpansive mappings.

Definition 5. $\left\{T_{i}\right\}_{i=1}^{m},\left\{S_{i}\right\}_{i=1}^{m}$ are said to satisfy condition $(B)$ if there exists a nondecreasing function $f:[0, \infty) \rightarrow[0, \infty)$ with $f(0)=0, f(r)>0$ for all $r \in(0, \infty)$, such that $\max _{1 \leq i \leq m}\left\{\left\|x-T_{i} x\right\|,\left\|x-S_{i} x\right\|\right\} \geq f(d(x, F))$ for all $x \in K$, where $F=\bigcap_{i=1}^{m} F\left(T_{i}\right) \cap F\left(S_{i}\right) \neq \emptyset$ and $d(x, F)=\inf _{x^{*} \in F} \| x-$ $x^{*} \|$.

\section{Weak Convergence Theorems for Asymptotically Quasi-Nonexpansive Mappings in Banach Spaces}

Lemma 6 (see [4]). Let $\left\{a_{n}\right\}_{n=1}^{\infty}$ and $\left\{l_{n}\right\}_{n=1}^{\infty}$ be two nonnegative real sequences satisfying

$$
a_{n+1} \leq\left(1+l_{n}\right) a_{n}, \quad \forall n \in \mathbb{N}
$$

where $\sum_{n=1}^{\infty} l_{n}<+\infty$; then $\lim _{n \rightarrow \infty} a_{n}$ exists.

Lemma 7 (see [5]). Let $p>1, r>0$ be two fixed numbers and let $E$ be a Banach space. Then $E$ is uniformly convex if and only if there exists a continuous, strictly increasing, and convex function $g:[0, \infty) \rightarrow[0, \infty)$ with $g(0)=0$ such that $\|\lambda x+(1-\lambda) y\|^{p} \leq \lambda\|x\|^{p}+(1-\lambda)\|y\|^{p}-\omega_{p}(\lambda) g(\|x-y\|)$ for all $x, y \in B_{r}(0)=\{x \in E:\|x\| \leq r\}$, and $\lambda \in[0,1]$, where $\omega_{p}(\lambda)=\lambda(1-\lambda)^{p}+(1-\lambda) \lambda^{p}$.

Lemma 8 (see [6]). Let $K$ be a nonempty closed subset of a uniformly convex Banach space $E$, and let $T: K \rightarrow K$ be an asymptotically nonexpansive mapping. Then $I-T$ is demiclosed at zero; that is, for each sequence $\left\{x_{n}\right\} \subset K$, if $\left\{x_{n}\right\}$ converges weakly to $p \in K$ and $\left\{(I-T) x_{n}\right\}$ converges strongly to 0 , then $p \in F(T)$.

Lemma 9 (see [7]). Let $E$ be a Banach space which satisfies Opial's condition and let $\left\{x_{n}\right\}$ be a sequence in $E$. Let $u, v \in E$ be such that $\lim _{n \rightarrow \infty}\left\|x_{n}-u\right\|$ and $\lim _{n \rightarrow \infty}\left\|x_{n}-v\right\|$ exist. If $\left\{x_{n_{k}}\right\}$ and $\left\{x_{m_{k}}\right\}$ are subsequences of $\left\{x_{n}\right\}$ which converge weakly to $u$ and $v$, respectively, then $u=v$.

Lemma 10. Let $K$ be a nonempty closed convex subset of a Banach space $E$, and let $\left\{T_{i}\right\}_{i=1}^{m},\left\{S_{i}\right\}_{i=1}^{m}$ be two finite families of asymptotically quasi-nonexpansive self-mappings of $K$ with sequences $\left\{u_{n}^{i}\right\}_{i=1}^{m},\left\{v_{n}^{i}\right\}_{i=1}^{m} \subset[0, \infty)$, and $F=\bigcap_{i=1}^{m} F\left(T_{i}\right) \cap$ $F\left(S_{i}\right) \neq \emptyset$. Let $\left\{x_{n}\right\}$ be the sequence defined by (4), if the following conditions are satisfied:

(i) $\sum_{n=1}^{\infty} u_{n}^{i}<+\infty, \sum_{n=1}^{\infty} v_{n}^{i}<+\infty$;

(ii) $\left\|x-T_{i} y\right\| \leq\left\|S_{i} x-T_{i} y\right\|$ for all $x, y \in K$ and $i=$ $1,2, \ldots, m$.

Then $\lim _{n \rightarrow \infty}\left\|x_{n}-T_{i} x_{n}\right\|=0$ and $\lim _{n \rightarrow \infty}\left\|x_{n}-S_{i} x_{n}\right\|=0$. 
Abstract and Applied Analysis

3

Proof. Let $x^{*} \in F, v_{n}=\max _{1 \leq i \leq m}\left\{u_{n}^{i}, v_{n}^{i}\right\}$ for each $n$. Since $\sum_{n=1}^{\infty} u_{n}^{i}<+\infty, \sum_{n=1}^{\infty} v_{n}^{i}<+\infty$ for each $i, \sum_{n=1}^{\infty} v_{n}<+\infty$.

Step 1. We prove that, for all $x^{*} \in F$ and $i=1,2, \ldots, m-$ $1, \lim _{n \rightarrow \infty}\left\|x_{n}-x^{*}\right\|$ and $\lim _{n \rightarrow \infty}\left\|y_{n}^{i}-x^{*}\right\|$ are existent and equal.

It follows from (4) that we obtain that for any $i=$ $1,2, \ldots, m-2$

$$
\begin{aligned}
&\left\|y_{n-q_{i}}^{i}-x^{*}\right\| \\
&=\left\|\left(1-\beta_{n}^{i+1}\right) S_{i+1}^{n} x_{n}+\beta_{n}^{i+1} T_{i+1}^{n} y_{n-q_{i+1}}^{i+1}-x^{*}\right\| \\
& \leq\left(1-\beta_{n}^{i+1}\right)\left\|S_{i+1}^{n} x_{n}-x^{*}\right\|+\beta_{n}^{i+1}\left\|T_{i+1}^{n} y_{n-q_{i+1}}^{i+1}-x^{*}\right\| \\
& \leq\left(1-\beta_{n}^{i+1}\right)\left(1+v_{n}\right)\left\|x_{n}-x^{*}\right\| \\
&+\beta_{n}^{i+1}\left(1+v_{n}\right)\left\|y_{n-q_{i+1}}^{i+1}-x^{*}\right\| \\
&=\left(1+v_{n}\right) \\
& \times\left(\left(1-\beta_{n}^{i+1}\right)\left\|x_{n}-x^{*}\right\|+\beta_{n}^{i+1}\left\|y_{n-q_{i+1}}^{i+1}-x^{*}\right\|\right),
\end{aligned}
$$

and for $i=m-1$, we have

$$
\begin{aligned}
&\left\|y_{n-q_{m-1}}^{m-1}-x^{*}\right\| \\
&=\left\|\left(1-\beta_{n}^{m}\right) S_{m}^{n} x_{n}+\beta_{n}^{m} T_{m}^{n} x_{n}-x^{*}\right\| \\
& \leq\left(1-\beta_{n}^{m}\right)\left\|S_{m}^{n} x_{n}-x^{*}\right\|+\beta_{n}^{m}\left\|T_{m}^{n} x_{n}-x^{*}\right\| \\
& \leq\left(1-\beta_{n}^{m}\right)\left(1+v_{n}\right)\left\|x_{n}-x^{*}\right\| \\
&+\beta_{n}^{m}\left(1+v_{n}\right)\left\|x_{n}-x^{*}\right\| \\
&=\left(1+v_{n}\right)\left\|x_{n}-x^{*}\right\| .
\end{aligned}
$$

Then, from (4), (6) and (7), we get

$$
\begin{aligned}
&\left\|x_{n+1}-x^{*}\right\| \\
&=\left\|\left(1-\beta_{n}^{1}\right) S_{1}^{n} x_{n}+\beta_{n}^{1} T_{1}^{n} y_{n-q_{1}}^{1}-x^{*}\right\| \\
& \leq\left(1-\beta_{n}^{1}\right)\left\|S_{1}^{n} x_{n}-x^{*}\right\|+\beta_{n}^{1}\left\|T_{1}^{n} y_{n-q_{1}}^{1}-x^{*}\right\| \\
& \leq\left(1-\beta_{n}^{1}\right)\left(1+v_{n}\right)\left\|x_{n}-x^{*}\right\| \\
&+\beta_{n}^{1}\left(1+v_{n}\right)\left\|y_{n-q_{1}}^{1}-x^{*}\right\| \\
&=\left(1+v_{n}\right) \\
& \times\left(\left(1-\beta_{n}^{1}\right)\left\|x_{n}-x^{*}\right\|+\beta_{n}^{1}\left\|y_{n-q_{1}}^{1}-x^{*}\right\|\right) \\
& \leq\left(1+v_{n}\right)^{2} \\
& \times\left(\left(1-\beta_{n}^{1} \beta_{n}^{2}\right)\left\|x_{n}-x^{*}\right\|+\beta_{n}^{1} \beta_{n}^{2}\left\|y_{n-q_{2}}^{2}-x^{*}\right\|\right)
\end{aligned}
$$$$
\left\|y_{n-q_{i}}^{i}-x^{*}\right\|^{2}
$$$$
=\left\|\left(1-\beta_{n}^{i+1}\right) S_{i+1}^{n} x_{n}+\beta_{n}^{i+1} T_{i+1}^{n} y_{n-q_{i+1}}^{i+1}-x^{*}\right\|^{2}
$$$$
\leq\left(1-\beta_{n}^{i+1}\right)\left\|S_{i+1}^{n} x_{n}-x^{*}\right\|^{2}+\beta_{n}^{i+1}\left\|T_{i+1}^{n} y_{n-q_{i+1}}^{i+1}-x^{*}\right\|^{2}
$$$$
-\omega_{2}\left(\beta_{n}^{i+1}\right) g\left(\left\|T_{i+1}^{n} y_{n-q_{i+1}}^{i+1}-S_{i+1}^{n} x_{n}\right\|\right)
$$$$
\leq\left(1+v_{n}\right)^{2}
$$$$
\times\left(\left(1-\beta_{n}^{i+1}\right)\left\|x_{n}-x^{*}\right\|^{2}+\beta_{n}^{i+1}\left\|y_{n-q_{i+1}}^{i+1}-x^{*}\right\|^{2}\right)
$$$$
-2 \epsilon^{3} g\left(\left\|T_{i+1}^{n} y_{n-q_{i+1}}^{i+1}-S_{i+1}^{n} x_{n}\right\|\right) \text {, }
$$

$$
\begin{aligned}
& \text { for any } i=1,2, \ldots, m-2 \text { and } \\
& \qquad \begin{aligned}
&\left\|y_{n-q_{m-1}}^{m-1}-x^{*}\right\|^{2} \\
&=\left\|\left(1-\beta_{n}^{m}\right) S_{m}^{n} x_{n}+\beta_{n}^{m} T_{m}^{n} x_{n}-x^{*}\right\|^{2} \\
& \leq\left(1-\beta_{n}^{m}\right)\left\|x_{n}-x^{*}\right\|^{2}+\beta_{n}^{m}\left\|T_{m}^{n} x_{n}-x^{*}\right\|^{2} \\
& \quad-\omega_{2}\left(\beta_{n}^{m}\right) g\left(\left\|T_{m}^{n} x_{n}-S_{m}^{n} x_{n}\right\|\right) \\
& \leq\left(1+v_{n}\right)^{2}\left\|x_{n}-x^{*}\right\|^{2}-2 \epsilon^{3} g\left(\left\|T_{m}^{n} x_{n}-S_{m}^{n} x_{n}\right\|\right) .
\end{aligned}
\end{aligned}
$$

where $l_{n}=C_{m} v_{n}+C_{m}^{2} v_{n}^{2}+\cdots+C_{m} v_{n}$. Since $\sum_{n=1}^{\infty} l_{n}=$ , 6 , see that $\lim _{n \rightarrow \infty}\left\|y_{n}^{i}-x^{*}\right\|$ also exist for all $i=1,2, \ldots m-1$

$-T_{i} x_{n}=0$ and Since $E$ is uniformly convex Banach space, from 
From (9), we have

$$
\begin{aligned}
& 2 \epsilon^{3} g\left(\left\|T_{1}^{n} y_{n-q_{1}}^{1}-S_{1}^{n} x_{n}\right\|\right) \\
& \leq\left(1-\beta_{n}^{1}\right)\left(\left(1+v_{n}\right)^{2}\left\|x_{n}-x^{*}\right\|^{2}-\left\|x_{n+1}-x^{*}\right\|^{2}\right) \\
& \quad+\beta_{n}^{1}\left(\left(1+v_{n}\right)^{2}\left\|y_{n-q_{1}}^{1}-x^{*}\right\|^{2}-\left\|x_{n+1}-x^{*}\right\|^{2}\right) .
\end{aligned}
$$

Since $\lim _{n \rightarrow \infty}\left\|x_{n}-x^{*}\right\|$ and $\lim _{n \rightarrow \infty}\left\|y_{n}^{i}-x^{*}\right\|$ are existent and equal, we have

$$
\lim _{n \rightarrow \infty} g\left(\left\|T_{1}^{n} y_{n-q_{1}}^{1}-S_{1}^{n} x_{n}\right\|\right)=0
$$

Because $g$ is strictly increasing and continuous and $g(0)=0$,

$$
\left\|T_{1}^{n} y_{n-q_{1}}^{1}-S_{1}^{n} x_{n}\right\| \longrightarrow 0 \quad(\text { as } n \longrightarrow \infty)
$$

Further, similar to the computations above, using (10) and (11), we also can get for any $i=1,2, \ldots, m-2$

$$
\lim _{n \rightarrow \infty}\left\|T_{i}^{n} y_{n-q_{i}}^{i}-S_{i}^{n} x_{n}\right\|=0, \quad \lim _{n \rightarrow \infty}\left\|T_{m}^{n} x_{n}-S_{m}^{n} x_{n}\right\|=0 .
$$

Hence, for all $i=1,2, \ldots, m-1$, we can obtain

$$
\lim _{n \rightarrow \infty}\left\|T_{i}^{n} y_{n-q_{i}}^{i}-S_{i}^{n} x_{n}\right\|=0, \quad \lim _{n \rightarrow \infty}\left\|T_{m}^{n} x_{n}-S_{m}^{n} x_{n}\right\|=0 .
$$

Since $\left\|x-T_{i} y\right\| \leq\left\|S_{i} x-T_{i} y\right\|$ for all $x, y \in K$, we have $\| x-$ $T_{i}^{n} y\|\leq\| S_{i}^{n} x-T_{i}^{n} y \|$, and then

$$
\begin{array}{r}
\lim _{n \rightarrow \infty}\left\|T_{i}^{n} y_{n-q_{i}}^{i}-x_{n}\right\| \leq \lim _{n \rightarrow \infty}\left\|T_{i}^{n} y_{n-q_{i}}^{i}-S_{i}^{n} x_{n}\right\|=0, \\
\forall i=1,2, \ldots, m-1 \\
\lim _{n \rightarrow \infty}\left\|T_{m}^{n} x_{n}-x_{n}\right\| \leq \lim _{n \rightarrow \infty}\left\|T_{m}^{n} x_{n}-S_{m}^{n} x_{n}\right\|=0 .
\end{array}
$$

From (16) and (17), for $i=1,2, \ldots, m-1$

$$
\begin{aligned}
&\left\|S_{i}^{n} x_{n}-x_{n}\right\| \leq\left\|S_{i}^{n} x_{n}-T_{i}^{n} y_{n-q_{i}}^{i}\right\|+\left\|T_{i}^{n} y_{n-q_{1}}^{i}-x_{n}\right\| \longrightarrow 0 \\
&\left\|S_{m}^{n} x_{n}-x_{n}\right\| \leq\left\|S_{m}^{n} x_{n}-T_{m}^{n} x_{n}\right\|+\left\|T_{m}^{n} x_{n}-x_{n}\right\| \\
& \longrightarrow 0 \quad(\text { as } n \rightarrow \infty),
\end{aligned}
$$

so

$$
\begin{aligned}
& \left\|x_{n+1}-x_{n}\right\| \\
& \quad=\left\|\left(1-\beta_{n}^{1}\right) S_{1}^{n} x_{n}+\beta_{n}^{1} T_{1}^{n} y_{n-q_{1}}^{1}-x_{n}\right\| \\
& \quad \leq\left(1-\beta_{n}^{1}\right)\left\|S_{1}^{n} x_{n}-x_{n}\right\|+\beta_{n}^{1}\left\|T_{1}^{n} y_{n-q_{1}}^{1}-x_{n}\right\| \\
& \quad \longrightarrow 0 \quad(\text { as } n \longrightarrow \infty) .
\end{aligned}
$$

Hence from (16) and (18), for $i=1,2, \ldots, m-2$, we have

$$
\begin{aligned}
& \left\|x_{n}-y_{n-q_{i}}^{i}\right\| \\
& =\left\|x_{n}-\left(\left(1-\beta_{n}^{i+1}\right) S_{i+1}^{n} x_{n}+\beta_{n}^{i+1} T_{i+1}^{n} y_{n-q_{i+1}}^{i+1}\right)\right\| \\
& \leq\left\|x_{n}-S_{i+1}^{n} x_{n}\right\|+\beta_{n}^{i+1}\left\|S_{i+1}^{n} x_{n}-T_{i+1}^{n} y_{n-q_{i+1}}^{i+1}\right\| \\
& \longrightarrow 0, \\
& \left\|x_{n}-y_{n-q_{m-1}}^{m-1}\right\| \\
& \quad=\left\|x_{n}-\left(\left(1-\beta_{n}^{m}\right) S_{m}^{n} x_{n}+\beta_{n}^{m} T_{m}^{n} x_{n}\right)\right\| \\
& \quad \leq\left\|x_{n}-S_{m}^{n} x_{n}\right\|+\beta_{n}^{m}\left\|S_{m}^{n} x_{n}-T_{m}^{n} x_{n}\right\| \longrightarrow 0
\end{aligned}
$$

$($ as $n \longrightarrow \infty)$.

It follows from (17) and (20) that, for $i=1,2, \ldots, m-1$,

$$
\begin{aligned}
& \left\|T_{i}^{n} x_{n}-x_{n}\right\| \\
& \quad \leq\left\|T_{i}^{n} x_{n}-T_{i}^{n} y_{n-q_{i}}^{i}\right\|+\left\|T_{i}^{n} y_{n-q_{i}}^{i}-x_{n}\right\| \\
& \quad \leq\left(1+v_{n}\right)\left\|x_{n}-y_{n-q_{i}}^{i}\right\|+\left\|T_{i}^{n} y_{n-q_{i}}^{i}-x_{n}\right\| \longrightarrow 0
\end{aligned}
$$

(as $n \longrightarrow \infty)$.

Together with (17), for $i=1,2, \ldots, m$

$$
\left\|T_{i}^{n} x_{n}-x_{n}\right\| \longrightarrow 0 \quad(\text { as } n \longrightarrow \infty) .
$$

From (19) and (22), for any $i=1,2, \ldots, m$, we have

$$
\begin{aligned}
& \left\|x_{n}-T_{i} x_{n}\right\| \\
& \leq\left\|x_{n}-x_{n+1}\right\|+\left\|x_{n+1}-T_{i}^{n+1} x_{n+1}\right\| \\
& +\left\|T_{i}^{n+1} x_{n+1}-T_{i}^{n+1} x_{n}\right\|+\left\|T_{i}^{n+1} x_{n}-T_{i} x_{n}\right\| \\
& \leq\left\|x_{n}-x_{n+1}\right\|+\left\|x_{n+1}-T_{i}^{n+1} x_{n+1}\right\| \\
& \quad+\left(1+v_{n+1}\right)\left\|x_{n+1}-x_{n}\right\|+\left(1+v_{1}\right)\left\|T_{i}^{n} x_{n}-x_{n}\right\| \\
& =\left(2+v_{n+1}\right)\left\|x_{n}-x_{n+1}\right\|+\left\|x_{n+1}-T_{i}^{n+1} x_{n+1}\right\| \\
& \quad+\left(1+v_{1}\right)\left\|T_{i}^{n} x_{n}-x_{n}\right\| \longrightarrow 0
\end{aligned}
$$$$
\text { (as } n \longrightarrow \infty) \text {. }
$$

Together with (16) and (20), for $i=1,2, \ldots, m-1$, we have

$$
\begin{aligned}
& \left\|S_{i}^{n} x_{n}-T_{i}^{n} x_{n}\right\| \\
& \quad \leq\left\|S_{i}^{n} x_{n}-T_{i}^{n} y_{n-q_{i}}^{i}\right\|+\left\|T_{i}^{n} y_{n-q_{i}}^{i}-T_{i}^{n} x_{n}\right\| \\
& \quad \leq\left\|S_{i}^{n} x_{n}-T_{i}^{n} y_{n-q_{i}}^{i}\right\|+\left(1+v_{n}\right)\left\|y_{n-q_{i}}^{i}-x_{n}\right\| \longrightarrow 0
\end{aligned}
$$

(as $n \longrightarrow \infty)$.

Together with (16), for $i=1,2, \ldots, m$, we have

$$
\left\|S_{i}^{n} x_{n}-T_{i}^{n} x_{n}\right\| \longrightarrow 0 \quad(\text { as } n \longrightarrow \infty) \text {. }
$$


Since $\left\|S_{i} x_{n}-T_{i}^{n} x_{n}\right\| \leq\left\|S_{i}^{n} x_{n}-T_{i}^{n} x_{n}\right\|$, together with (23) and (25), for $i=1,2, \ldots, m$, then

$$
\begin{aligned}
& \left\|x_{n}-S_{i} x_{n}\right\| \\
& \leq\left\|x_{n}-T_{i}^{n} x_{n}\right\|+\left\|T_{i}^{n} x_{n}-S_{i} x_{n}\right\| \\
& \leq\left\|x_{n}-T_{i}^{n} x_{n}\right\|+\left\|T_{i}^{n} x_{n}-S_{i}^{n} x_{n}\right\| \longrightarrow 0 \\
& \quad(\text { as } n \longrightarrow \infty) .
\end{aligned}
$$

Theorem 11. Under the assumptions of Lemma 10, if $E$ is a uniformly convex Banach space satisfying Opial's condition, then the sequence $\left\{x_{n}\right\}_{n=1}^{\infty}$ given by (4) converges weakly to a common fixed point of $\left\{T_{i}\right\}_{i=1}^{m}$ and $\left\{S_{i}\right\}_{i=1}^{m}$.

Proof. By using the same proof as in Lemma 10, it can be shown that for any $i=1,2, \ldots, m$

$$
\lim _{n \rightarrow \infty}\left\|x_{n}-T_{i} x_{n}\right\|=0, \quad \lim _{n \rightarrow \infty}\left\|x_{n}-S_{i} x_{n}\right\|=0
$$

So $I-T_{i}$ and $I-S_{i}$ are demiclosed at 0 .

Since $E$ is uniformly convex and $\left\{x_{n}\right\}$ is bounded, we may assume that $x_{n} \rightarrow u$ as $n \rightarrow \infty$, without loss of generality. By Lemma 8 , we have $u \in F$. Suppose that subsequences $\left\{x_{n_{k}}\right\}$ and $\left\{x_{m_{k}}\right\}$ of $\left\{x_{n}\right\}$ converge weakly to $u$ and $v$, respectively. From Lemma $8, u, v \in F$. By Lemma $10, \lim _{n \rightarrow \infty}\left\|x_{n}-u\right\|$ and $\lim _{n \rightarrow \infty}\left\|x_{n}-v\right\|$ exist. It follows from Lemma 9 that $u=v$. Therefore $\left\{x_{n}\right\}$ converges weakly to a common fixed point of $\left\{T_{i}\right\}_{i=1}^{m}$ and $\left\{S_{i}\right\}_{i=1}^{m}$.

\section{Strong Convergence Theorems for Asymptotically Quasi-Nonexpansive Mappings in Banach Spaces}

In this section, we prove strong convergence theorems of the iterative schemes (4) in Banach spaces.

Theorem 12. Under the assumptions of Lemma 10, the sequence $\left\{x_{n}\right\}_{n=1}^{\infty}$ given by (4) converges strongly to $x^{\prime} \in F$ if and only if $\liminf _{n \rightarrow \infty} d\left(x_{n}, F\right)=0$, where $d\left(x_{n}, F\right)=$ $\inf _{p \in F}\left\|x_{n}-p\right\|$.

Proof. Necessity is obvious. We only prove the sufficiency. Suppose that $\liminf _{n \rightarrow \infty} d\left(x_{n}, F\right)=0$. As proved in Lemma 10, for each $x^{*} \in F$, we have $\left\|x_{n+1}-x^{*}\right\| \leq(1+$ $\left.l_{n}\right)\left\|x_{n}-x^{*}\right\|$; that is, $d\left(x_{n+1}, F\right) \leq\left(1+l_{n}\right) d\left(x_{n}, F\right)$. From Lemma $6, \lim _{n \rightarrow \infty} d\left(x_{n}, F\right)$ exists, based on the assumption that $\lim _{n \rightarrow \infty} d\left(x_{n}, F\right)=0$.

Next, we can prove that $\left\{x_{n}\right\}$ is a Cauchy sequence in $K$. In fact, for any $x^{*} \in F$,

$$
\left\|x_{n+1}-x^{*}\right\| \leq\left(1+l_{n}\right)\left\|x_{n}-x^{*}\right\|, \quad \forall n \geq 1
$$

where $\sum_{n=1}^{\infty} l_{n}<+\infty$. Hence for any positive integers $n, m$, we have

$$
\begin{aligned}
& \left\|x_{n+m}-x_{n}\right\| \\
& \quad \leq\left\|x_{n+m}-x^{*}\right\|+\left\|x_{n}-x^{*}\right\| \\
& \quad \leq\left(1+l_{n+m-1}\right)\left\|x_{n+m-1}-x^{*}\right\|+\left\|x_{n}-x^{*}\right\| .
\end{aligned}
$$

Since $x \geq 0$, then $1+x \leq e^{x}$. Thus, we get

$$
\begin{aligned}
& \left\|x_{n+m}-x_{n}\right\| \\
& \quad \leq\left\|x_{n+m}-x^{*}\right\|+\left\|x_{n}-x^{*}\right\| \\
& \quad \leq e^{l_{n+m-1}}\left\|x_{n+m-1}-x^{*}\right\|+\left\|x_{n}-x^{*}\right\| \\
& \leq e^{l_{n+m-1}+l_{n+m-2}}\left\|x_{n+m-2}-x^{*}\right\|+\left\|x_{n}-x^{*}\right\| \\
& \quad \vdots \\
& \quad \leq e^{\sum_{i=n}^{n+m-1} l_{i}}\left\|x_{n}-x^{*}\right\|+\left\|x_{n}-x^{*}\right\| \\
& \leq(1+M)\left\|x_{n}-x^{*}\right\|,
\end{aligned}
$$

where $M=e^{\sum_{i=1}^{\infty} l_{i}}<\infty$. So we have

$$
\left\|x_{n+m}-x_{n}\right\| \leq(1+M)\left\|x_{n}-x^{*}\right\|
$$

This shows that $\left\{x_{n}\right\}$ is a Cauchy sequence in $K$, since $K$ is a nonempty closed convex subset of a Banach space $E$; that is, $K$ is a complete space. Without loss of generality, we can assume that $\left\{x_{n}\right\}$ converges strongly to a common fixed point $p \in F$.

Theorem 13. Under the assumptions of Lemma 10, if $\left\{T_{i}\right\}_{i=1}^{m},\left\{S_{i}\right\}_{i=1}^{m}$ satisfy condition (B), then the sequence $\left\{x_{n}\right\}$ defined by (4) converges strongly to a common fixed point $x^{*} \in F$.

Proof. It follows from Lemma 10 that, for any $i=1,2, \ldots, m$, we have

$$
\lim _{n \rightarrow \infty}\left\|x_{n}-T_{i} x_{n}\right\|=0, \quad \lim _{n \rightarrow \infty}\left\|x_{n}-S_{i} x_{n}\right\|=0 .
$$

Since $\left\{T_{i}\right\}_{i=1}^{m},\left\{S_{i}\right\}_{i=1}^{m}$ satisfy condition (B), we have $\lim _{n \rightarrow \infty} f\left(d\left(x_{n}, F\right)\right)=0$.

Since $f$ is a nondecreasing function with $f(0)=0, f(r)>$ 0 for all $r>0$, such that, for all $x_{n} \in E, \lim _{n \rightarrow \infty} d\left(x_{n}, F\right)=0$; by Theorem 12, we obtain that $\left\{x_{n}\right\}$ converges strongly to a common fixed point $x^{*} \in F$. 
Corollary 14. Under the assumptions of Lemma 10, the iteration sequence $\left\{x_{n}\right\}$ is defined as follows:

$$
\begin{gathered}
x_{j} \in E \quad(j=1,2, \ldots r), \\
x_{n+1}=\left(1-\beta_{n}^{1}\right) S_{1}^{n} x_{n}+\beta_{n}^{1} T_{1}^{n} y_{n-1}^{1}, \\
y_{n-i}^{i}=\left(1-\beta_{n}^{i+1}\right) S_{i+1}^{n} x_{n}+\beta_{n}^{i+1} T_{i+1}^{n} y_{n-(i+1)}^{i+1}, \\
i=1,2, \ldots, m-2, \\
y_{n-(m-1)}^{m-1}=\left(1-\beta_{n}^{m}\right) S_{m}^{n} x_{n}+\beta_{n}^{m} T_{m}^{n} x_{n}, \\
2 \leq m \leq r, \quad n \geq r,
\end{gathered}
$$

where $\left\{\beta_{n}^{i}\right\}_{i=1}^{m} \subset[\epsilon, 1-\epsilon]$ with $\epsilon \in(0,1)$.

(i) If $E$ satisfies Opial's condition, then $\left\{x_{n}\right\}$ converges weakly to a common fixed point $x^{*} \in F$.

(ii) If $\left\{T_{i}\right\}_{i=1}^{m},\left\{S_{i}\right\}_{i=1}^{m}$ satisfy condition $(B)$, then $\left\{x_{n}\right\}$ converges strongly to a common fixed point of $x^{*} \in F$.

Proof. By taking $\left\{q_{i}\right\}_{i=1}^{m}=i$ for all $n \geq 1$ in (4), from Theorems 11 and 13, the conclusion of the corollary follows.

Corollary 15. Under the assumptions of Lemma 10, the iteration sequence $\left\{x_{n}\right\}$ is defined as follows:

$$
\begin{gathered}
x_{1} \in E \\
x_{n+1}=\left(1-\beta_{n}^{1}\right) S_{1}^{n} x_{n}+\beta_{n}^{1} T_{1}^{n} y_{n}^{1}, \\
y_{n}=\left(1-\beta_{n}^{i+1}\right) S_{i+1}^{n} x_{n}+\beta_{n}^{i+1} T_{i+1}^{n} y_{n}^{i+1}, \\
i=1,2, \ldots, m-2, \\
y_{n}^{m-1}=\left(1-\beta_{n}^{m}\right) S_{m}^{n} x_{n}+\beta_{n}^{m} T_{m}^{n} x_{n}, \\
m \geq 2,
\end{gathered}
$$

where $\left\{\beta_{n}^{i}\right\}_{i=1}^{m} \subset[\epsilon, 1-\epsilon]$ with $\epsilon \in(0,1)$.

(i) If $E$ satisfies Opial's condition, then $\left\{x_{n}\right\}$ converges weakly to a common fixed point $x^{*} \in F$.

(ii) If $\left\{T_{i}\right\}_{i=1}^{m},\left\{S_{i}\right\}_{i=1}^{m}$ satisfy condition $(B)$, then $\left\{x_{n}\right\}$ converges strongly to a common fixed point of $x^{*} \in F$.

Proof. By taking $r=1, q_{i}=0$ for all $i=1,2, \ldots, m$ in (4), from Theorems 11 and 13, the conclusion of the corollary follows. This completes the proof.

Corollary 16. Let $K$ be a nonempty closed convex subset of a uniformly convex Banach space $E$, and let $\left\{T_{i}\right\}_{i=1}^{m}$ be a family of asymptotically quasi-nonexpansive self-mappings of $K$ with sequences $\left\{u_{n}^{i}\right\}_{i=1}^{m} \subset[0, \infty)$ such that $\sum_{n=1}^{\infty} u_{n}^{i}<+\infty$ and $F=\bigcap_{i=1}^{m} F\left(T_{i}\right) \neq \emptyset$. The iteration sequence $\left\{x_{n}\right\}$ is defined by (2) satisfying $\left\{\beta_{n}^{i}\right\}_{i=1}^{m} \subset[\epsilon, 1-\epsilon]$ with $\epsilon \in(0,1)$.

(i) If $E$ satisfies Opial's condition, then $\left\{x_{n}\right\}$ converges weakly to a common fixed point of $\left\{T_{i}\right\}_{i=1}^{m}$. (ii) If $\left\{T_{i}\right\}_{i=1}^{m}$ satisfies condition $(B)$, then $\left\{x_{n}\right\}$ converges strongly to a common fixed point of $\left\{T_{i}\right\}_{i=1}^{m}$.

Proof. By taking $r=1,\left\{S_{i}\right\}_{i=1}^{m}=I$, and $y_{n-q_{i+1}}^{i+1}=y_{n+m-(i+2)}$ for all $i=0,1, \ldots, m-2$ in (4), we get (3). From Theorems 11 and 13 , the conclusion of the corollary follows.

Corollary 17. Let $K$ be a nonempty closed convex subset of a uniformly convex Banach space $E$, and let $\left\{T_{i}\right\}_{i=1}^{m}$ be a family of nonexpansive self-mappings of $K$ with sequences $\left\{u_{n}^{i}\right\}_{i=1}^{m} \subset$ $[0, \infty)$ such that $\sum_{n=1}^{\infty} u_{n}^{i}<+\infty$ and $F=\bigcap_{i=1}^{m} F\left(T_{i}\right) \neq \emptyset$. The iteration sequence $\left\{x_{n}\right\}$ is defined by (2) satisfying $\left\{\beta_{n}^{i}\right\}_{i=1}^{m} \subset$ $[\epsilon, 1-\epsilon]$ with $\epsilon \in(0,1)$.

(i) If E satisfies Opial's condition, then $\left\{x_{n}\right\}$ converges weakly to a common fixed point of $\left\{T_{i}\right\}_{i=1}^{m}$.

(ii) If $\left\{T_{i}\right\}_{i=1}^{m}$ satisfies condition $(B)$, then $\left\{x_{n}\right\}$ converges strongly to a common fixed point of $\left\{T_{i}\right\}_{i=1}^{m}$.

Proof. By taking $r=1,\left\{T_{i}^{n}\right\}_{i=1}^{m}=T,\left\{S_{i}\right\}_{i=1}^{m}=I$, and $\left\{q_{i}\right\}_{i=1}^{m}=0$ for all $n \geq 1$ in (4), we get (2), which was introduced by Rhoades and Soltuz in [2]. From Theorems 11 and 13, the conclusion of the corollary follows.

\section{Conflict of Interests}

The authors declare that there is no conflict of interests regarding the publication of this paper.

\section{Acknowledgments}

This work is supported by the National Natural Science Foundation of China (11226228), the Science and Technology Program Project of Henan Province (122300410256), and the Natural Science Foundation of the Education Department of Henan Province (2011B110025).

\section{References}

[1] K. Goebel and W. A. Kirk, "A fixed point theorem for asymptotically nonexpansive mappings," Proceedings of the American Mathematical Society, vol. 35, pp. 171-174, 1972.

[2] B. E. Rhoades and S. M. Soltuz, "The equivalence between Mann-Ishikawa iterations and multistep iteration," Nonlinear Analysis. Theory, Methods \& Applications, vol. 58, no. 1-2, pp. 219-228, 2004.

[3] C. E. Chidume and B. Ali, "Weak and strong convergence theorems for finite families of asymptotically nonexpansive mappings in Banach spaces," Journal of Mathematical Analysis and Applications, vol. 330, no. 1, pp. 377-387, 2007.

[4] S. S. Chang, H. W. Joseph Lee, and C. K. Chan, "On Reich's strong convergence theorem for asymptotically nonexpansive mappings in Banach spaces," Nonlinear Analysis. Theory, Methods \& Applications, vol. 66, no. 11, pp. 2364-2374, 2007.

[5] H. K. Xu, "Inequalities in Banach spaces with applications," Nonlinear Analysis. Theory, Methods \& Applications, vol. 16, no. 12, pp. 1127-1138, 1991.

[6] J. Schu, "Weak and strong convergence to fixed points of asymptotically nonexpansive mappings," Bulletin of the Australian Mathematical Society, vol. 43, no. 1, pp. 153-159, 1991. 
[7] S. Suantai, "Weak and strong convergence criteria of Noor iterations for asymptotically nonexpansive mappings," Journal of Mathematical Analysis and Applications, vol. 311, no. 2, pp. 506-517, 2005.

[8] M. O. Osilike and S. C. Aniagbosor, "Weak and strong convergence theorems for fixed points of asymptotically nonexpansive mappings," Mathematical and Computer Modelling, vol. 32, no. 10, pp. 1181-1191, 2000.

[9] Y. Zhou and S.-S. Chang, "Convergence of implicit iteration process for a finite family of asymptotically nonexpansive mappings in Banach spaces," Numerical Functional Analysis and Optimization, vol. 23, no. 7-8, pp. 911-921, 2002.

[10] C. E. Chidume, E. U. Ofoedu, and H. Zegeye, "Strong and weak convergence theorems for asymptotically nonexpansive mappings," Journal of Mathematical Analysis and Applications, vol. 280, no. 2, pp. 364-374, 2003.

[11] C. E. Chidume and N. Shahzad, "Strong convergence of an implicit iteration process for a finite family of nonexpansive mappings," Nonlinear Analysis. Theory, Methods \& Applications, vol. 62, no. 6, pp. 1149-1156, 2005. 


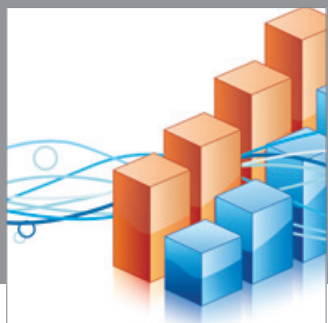

Advances in

Operations Research

mansans

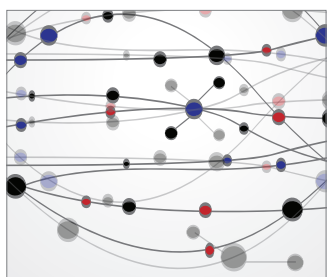

The Scientific World Journal
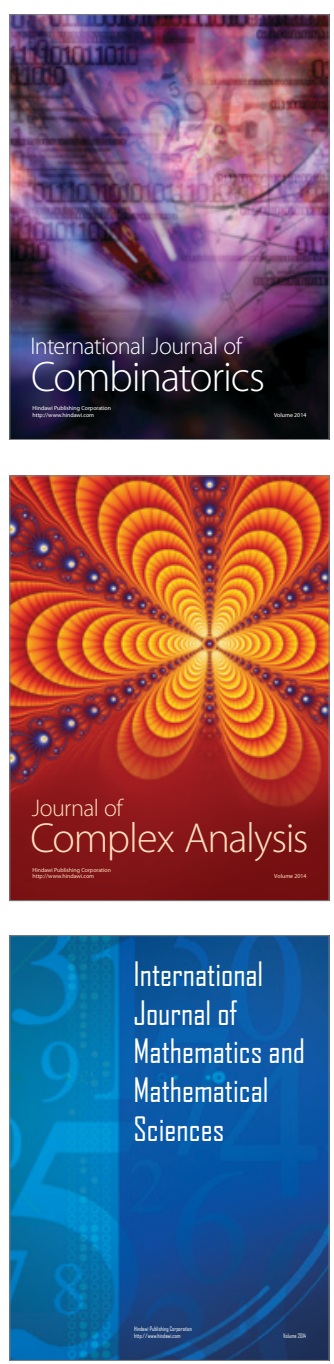
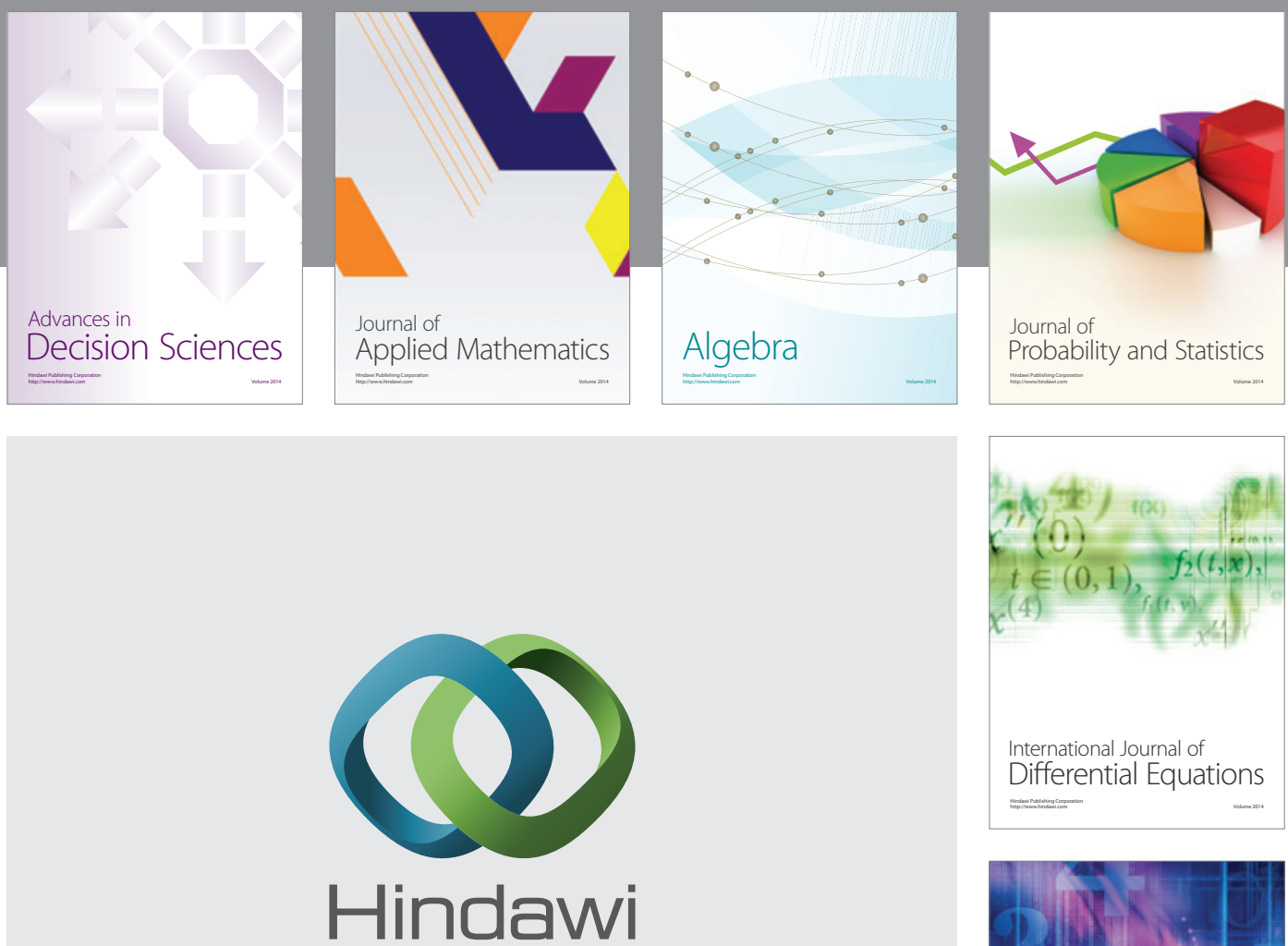

Submit your manuscripts at http://www.hindawi.com
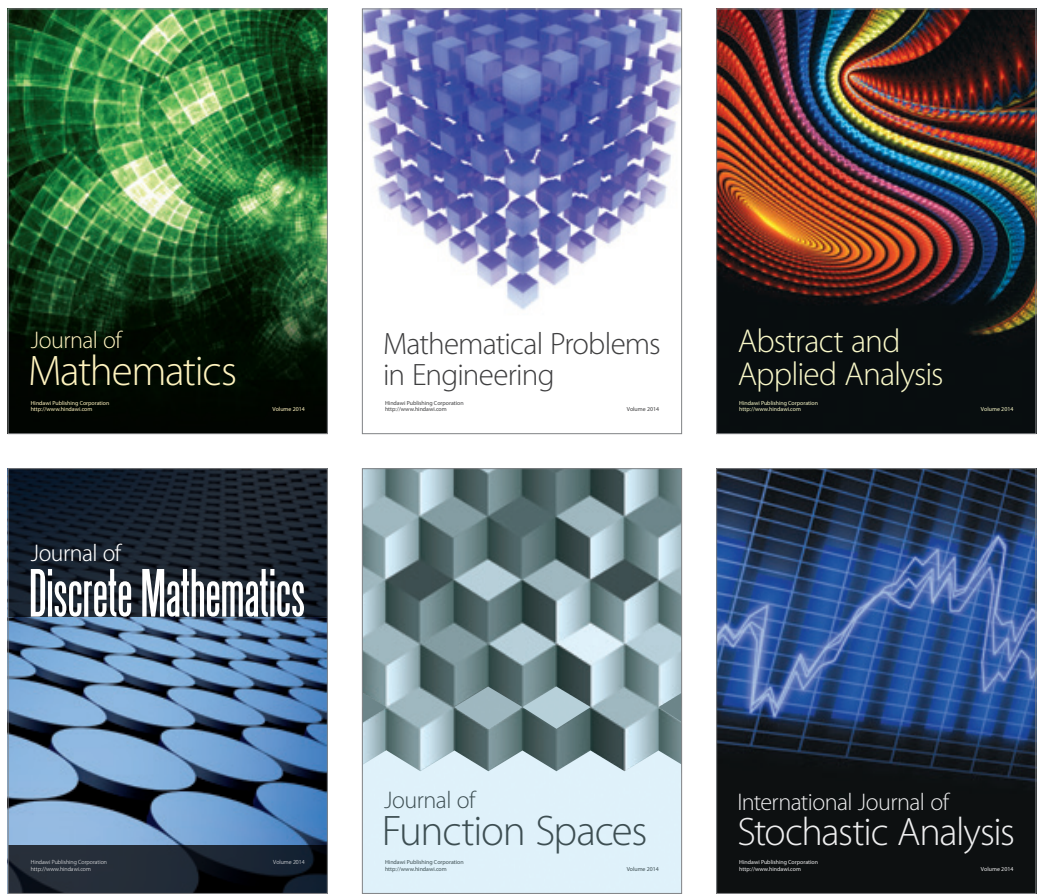

Journal of

Function Spaces

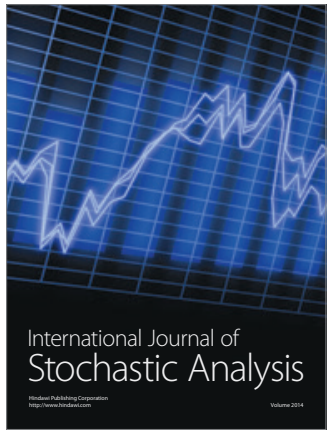

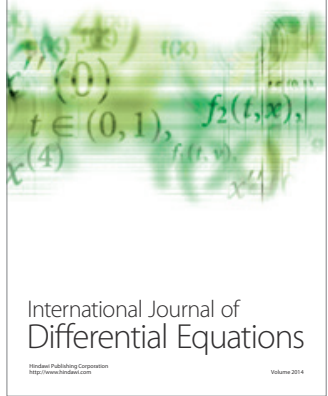
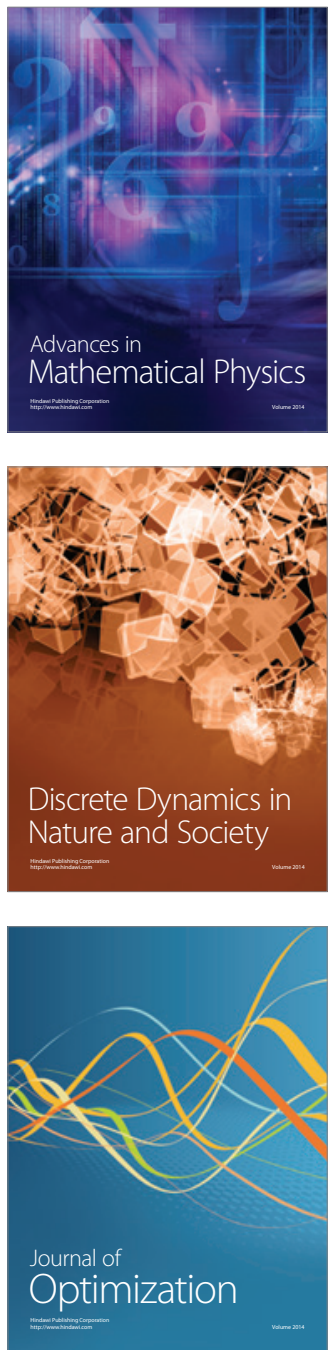